\title{
Co-culture of the bone and bone marrow: a novel way to obtain mesenchymal stem cells with enhanced osteogenic ability for fracture healing in SD rats
}

Cong Zhu', Mo Sha ${ }^{1}$, Huixiang Jiang ${ }^{2}$, Jianbiao Lin', Weibin Lin', Wenchang Li ${ }^{2}$ Xiaoshan Chen ${ }^{1}$, Guofeng Huang $^{1 *}$ (D) and Zhenqi Ding ${ }^{1 *}$

\begin{abstract}
Background: Mesenchymal stem cells (MSCs) have great potential for the repair and regeneration of bone fracture, but their optimal origins remain controversial.

Methods: Bone marrow-MSCs (BM-MSCs) and bone-bone marrow-MSCs (B-BM-MSCs) were isolated from 12 SD rats, and the morphology, MSC-associated markers, and proliferative capacity of these cells were compared using an inverted microscope, flow cytometry, and CCK-8 assays, respectively. After 14 days of osteoblastic induction, osteoblast phenotypes were detected by ALP and calcium nodule staining, and the expression of BMP-2 and TGF- $\beta 1$ was observed by western blotting. Then, the rat tibia fracture model was established with 3 groups $(n=6$ per group), the control, BM-MSC, and B-BM-MSC groups. Computed tomography (CT) imaging was performed to evaluate fracture healing at weeks 2, 4, and 6. Finally, the fractured bones were removed at weeks 4 and 6, and HE staining was performed to evaluate fracture healing.

Results: Although the 2 types of MSCs shared the same cellular morphology and MSC-associated markers, B-BMMSCs had a higher proliferative rate than BM-MSCs from day 9 to day $12(p<0.05)$, and the expression levels of ALP and calcium were obviously higher in B-BM-MSCs than in BM-MSCs after osteogenic induction $(p<0.01$ and $p<0.001$, respectively). Western blot results showed that the expression levels of BMP-2 and TGF- $\beta 1$ in B-BM-MSCs were higher than in BM-MSCs before and after osteogenic induction $(p<0.01)$. In the animal experiments, CT imaging and gross observation showed that B-BM-MSCs had a greater capacity than BM-MSCs to promote fracture healing, as the Lane-Sandhu scores of B-BM-MSCs at weeks 4 and 6 after operation (3.00 \pm 0.81 and $9.67 \pm 0.94$, respectively) were higher than those of BM-MSCs (1.33 \pm 0.47 and $6.67 \pm 1.25$, respectively; both $p<0.05)$. The HE staining results further supported this conclusion.
\end{abstract}

Conclusions: Taken together, our study results proved that MSCs obtained by co-culturing the bone and bone marrow from SD rats had better proliferative, osteogenic differentiation, and fracture healing capacities than BMMSCs, perhaps suggesting a novel way to obtain MSCs for bone tissue repair.

Keywords: Mesenchymal stem cells, Co-culture, Osteogenic differentiation, Bone fracture, Healing

\footnotetext{
*Correspondence: huangguofeng@xmu.edu.cn; dingzhenqi175@163.com ${ }^{1}$ Center for Orthopedics, Affiliated Southeast Hospital of Xiamen University/ 909th Hospital of People's Liberation Army, 269 Zhanghua Middle Road, Zhangzhou 363000, Fujian Province, China

Full list of author information is available at the end of the article
}

(C) The Author(s). 2019 Open Access This article is distributed under the terms of the Creative Commons Attribution 4.0 International License (http://creativecommons.org/licenses/by/4.0/), which permits unrestricted use, distribution, and reproduction in any medium, provided you give appropriate credit to the original author(s) and the source, provide a link to the Creative Commons license, and indicate if changes were made. The Creative Commons Public Domain Dedication waiver (http://creativecommons.org/publicdomain/zero/1.0/) applies to the data made available in this article, unless otherwise stated. 


\section{Introduction}

Fracture is a common surgical complication that is expensive to treat and has negative effects on individuals and society. In addition, approximately $10 \%$ of fractures cannot be cured in a normal way [1, 2]. Orthopedists have adopted many solutions to promote the regeneration of bone tissues, among which stem cell therapy [3-6] plays an important role. Mesenchymal stem cells (MSCs) are a type of adult stem cell that can develop into cells of bone, adipose, cartilage, tendon, ligament, et cetera $[7,8]$. MSCs are used as seed cells in tissue engineering transplantation because of their high proliferative capacity, multidirectional differentiation potential, low immunogenicity, and paracrine effects [9]. At present, the available sources of MSCs are the umbilical cords, bone marrow, dental pulp, bone, adipose, et cetera. However, there are currently no excellent methods for obtaining MSCs for fracture treatment have been found yet. For example, it is difficult to apply umbilical cord MSCs in clinical practice due to limited sources [10, 11]. For MSCs derived from the bone marrow (BM-MSCs), the bone marrow has a small number of MSCs and their osteogenic potential is weaker than that of bone MSCs (B-MSCs) [11, 12]. Similarly, adipose-derived MSCs have worse osteogenic potential than B-MSCs [13]. Although B-MSCs can be used as important seed cells for promoting bone regeneration, large amounts of the bone isolated from the body would cause serious secondary damage, severely limiting its clinical application [13, 14]. Other approaches to MSC acquisition also face challenges in sourcing, tumorigenicity control, osteogenic potential, et cetera $[15,16]$. Therefore, it is necessary to develop a new approach to extract MSCs with great proliferative capacity and osteogenic potential from various sources while causing minimal damage to the body.

Studies have shown that co-culture of cartilage and MSCs can improve the chondrogenic ability of MSCs $[17,18]$, and the stimulation of MSCs with fibroblast growth factor can enhance their ability to promote fracture repair of MSCs [19]. These results suggest that MSCs interact with the environment in ways that affect their growth. Meanwhile, the bone and bone marrow coexist in biological organisms, and MSCs in co-cultures of the bone and bone marrow are more similar to endogenous cells. In this study, we developed a novel way to obtain MSCs by co-culturing the bone and bone marrow (B-BM-MSCs) and explored whether the acquired MSCs are more effective at healing bone tissues.

To address this problem, 2 types of MSCs were isolated from the bone marrow and from co-cultures of the bone and bone marrow, and the cellular characteristics and capacity for fracture healing of the 2 types of cells were compared. Since TGF- $\beta 1$ and BMP- 2 play important regulatory roles in the osteogenic differentiation of mesenchymal stem cells [20, 21], we examined the expression of TGF- $\beta 1$ and BMP-2 before and after osteogenic induction in both groups and further explored the relevant mechanisms.

\section{Materials and methods}

Isolation and culture of rat B-BM-MSCs and BM-MSCs

A 6-week-old male SD rat was sacrificed with an injection of $10 \%$ chloral hydrate, and the femur and tibia were removed and placed into a sterile Petri dish.

(1) To obtain B-BM-MSCs, the medullary cavity was washed with PBS mixed with heparin sodium $(0.04 \mathrm{mg} /$ ml, H8270, Beijing Solarbio Science \& Technology Co., Ltd.) until it appeared clean of all periostea, and the total marrow isolate was collected by centrifugation. Then, the clean femur and tibia were cut into $3 \mathrm{~mm} \times 3 \mathrm{~mm}$ bone pellets and placed in a Petri dish with collagenase I (3 mg/mL, c8150, Beijing Solarbio Science \& Technology Co., Ltd.). The dish was incubated in a cell incubator (37 ${ }^{\circ} \mathrm{C}, 5 \% \mathrm{CO}_{2}$ ) for $45 \mathrm{~min}$ [19]. At the same time, the bone marrow sample with a cell concentration of $2 \times 10^{8} / \mathrm{mL}$ to $1 \times 10^{9} / \mathrm{mL}$ was resuspended in PBS, $5 \mathrm{~mL}$ rat mesenchymal cell separation fluid (LGS1072, Tianjin HaoYang HuaKe Biological Technology Co., Ltd.) was added to a $15 \mathrm{~mL}$ centrifuge tube, the cell suspension was placed onto the separation liquid surface, and the tube was centrifuged at $450 \times g$ for $30 \mathrm{~min}$ at room temperature. The second layer of the milky white cell layer was placed in another $15 \mathrm{~mL}$ centrifuge tube and washed twice with PBS. Then, the cells were filtered through a filter with a pore size of $74 \mu \mathrm{m}$, and the filtered liquid was collected into a $6-\mathrm{cm}$ Petri dish containing $6 \mathrm{~mL}$ MSC complete medium [DMEM/High Glucose (HyClone, USA) + 10\% FBS (No. 04-001-1A, Biological Industries, Israel) + 1\% streptomycin/penicillin (100× SV30010, HyClone, USA) + $50 \mu \mathrm{mol} / \mathrm{L} \beta$-mercaptoethanol (M8210, Beijing Solarbio Science \& Technology Co., Ltd.)]. After a 45-min incubation, 3 pieces of the bone were moved to the same Petri dish, and the dish was placed into a cell incubator. The medium was changed every $48 \mathrm{~h}$, and the cells were maintained in the same medium until they reached approximately $80 \%$ confluence. These cells were considered passage 0 . Cells were then trypsinized (0.5\% trypsin, T8150, Beijing Solarbio Science \& Technology Co., Ltd.) and re-cultured for the next passage.

(2) To obtain BM-MSCs, the cells in the bone marrow were obtained as mentioned above and cultured using the same approach as that for B-BM-MSCs. The isolation and culture of BM-MSCs were guided by the methods reported by Blashki [22].

Two types of P3 MSCs were transfected with lentivirus carrying the green fluorescent protein (GFP) gene (multiplicity of infection = 100). After $12 \mathrm{~h}$, the medium containing the virus solution was discarded, and MSC 
complete medium was added for 2 or 3 days. The cells were then photographed with a microscope. The sample size was 12 .

\section{Analysis of MSC-associated markers by flow cytometry} The P3 cells were harvested and adjusted to a concentration of $1 \times 10^{6} / \mathrm{mL}$ prior to staining with antibodies against CD90 (11-0900-81), CD44 (12-0444-80), CD29 (17-0291-80), CD45 (11-0461-80), CD31 (25-0310-80) (Thermo Fisher Scientific, Ebioscience, USA), and CD106 (lot 130-103-684, Miltenyi Biotec, Germany). Fluorescence-activated cell identification was performed with flow cytometry (Beckman USA), and the data were analyzed with CytExpert (Tree Star, Ashland, OR, USA). The sample size was 12 .

\section{Proliferation ability of MSCs from different sources}

After a 9-day cultivation, the concentration of the 2 types of MSCs was adjusted to $2 \times 10^{4}$ cells $/ \mathrm{mL}$, and these cell solutions inoculated in a 96-well cell culture plate with $100 \mu \mathrm{L} /$ well. After culture for 1,2 , 3, and 4 days, $10 \mu \mathrm{L}$ CCK-8 reagent (FC101-03, TransGen Biotech, Beijing, China) was added to each well, and the plates were incubated at $37{ }^{\circ} \mathrm{C}$ with $5 \% \mathrm{CO}_{2}$ for $2 \mathrm{~h}$. The OD values were determined at $450 \mathrm{~nm}$ with a microplate reader (Bio-Rad, USA). The cell proliferation rate was calculated as follows: rate $($ day $X)=[\mathrm{OD} 450($ day $X)$-OD450 (day $X$-1)]/OD450 (day $X-1)$. The sample size was 6.

\section{Osteogenic differentiation ability of MSCs from different sources}

The P3 MSCs from the 2 different sources were seeded in 6 -cm dishes with $2 \times 10^{5}$ cells per dish. The cells were cultured at $37{ }^{\circ} \mathrm{C}$ with $5 \% \mathrm{CO}_{2}$ for $24 \mathrm{~h}$. Then, the original medium was replaced with osteogenic induction medium containing $10^{-2} \mathrm{~mol} / \mathrm{L}$ sodium glycerophosphate (MB3195, Dalian Meilun Biotech Co., Ltd.), $10^{-7} \mathrm{~mol} / \mathrm{L}$ dexamethasone (MB1434, Dalian Meilun Biotech Co., Ltd.), and $3 \times 10^{-4} \mathrm{~mol} / \mathrm{L}$ vitamin C (MB3195, Dalian Meilun Biotech Co., Ltd.). The induction medium was changed every 2 days, and the cells were induced for 14 days. Then, the cells were fixed, ALP and calcium nodules were stained with the modified Gomori Calcium-Cobalt method (DE0001, Beijing Leagene Biotech Co., Ltd.), and alizarin red staining (DS0002, Beijing Leagene Biotech Co., Ltd.) was performed according to the manufacturer's instructions. The ratio of the positive area under each high-power field (RPA-HPT) was used to evaluate ALP expression and calcium nodules. The sample size was 6 .

\section{The expression of TGF- $\beta 1$ and BMP- 2 in the 2 types of MSCs before and after osteogenic induction}

Cell samples were isolated with RIPA buffer (RIPA:PMSF $=100: 1$, R0020 and P8340, Beijing Solarbio Science \&
Technology Co., Ltd), and the total protein content was measured with the bicinchoninic acid protein assay kit (Thermo Scientific, Waltham, MA, USA). After the addition of loading buffer, the samples were boiled for 5 min for protein denaturation. Then, the samples were separated by sodium dodecyl sulfate-polyacrylamide gel electrophoresis (SDS-PAGE) in a $12 \%$ gel under a constant voltage of $80 \mathrm{~V}$ for $30 \mathrm{~min}$ followed by a constant voltage of $110 \mathrm{~V}$ until the samples reached the bottom of the separation gel. Proteins were resolved by denaturing SDSPAGE followed by transfer onto a nitrocellulose membrane. GAPDH (AB0037, Shanghai Abways Biotechnology Co., Ltd.) was used as the loading control. The membranes were incubated overnight at $4{ }^{\circ} \mathrm{C}$ with primary antibodies against TGF- $\beta 1$ (1:1000, $\arg 10002$, Arigo Biolaboratories, Taiwan, China), BMP-2 (1:500, arg65980, Arigo Biolaboratories, Taiwan, China), and GAPDH (1: 5000). The membranes were incubated with secondary antibodies (HS101-01 and HS201-01, Beijing TransGen Biotech Co., Ltd.) conjugated with horseradish peroxidase for $1 \mathrm{~h}$. Target proteins were detected by an enhanced chemiluminescence system (4AWO12-050, Beijing 4A Biotech, Co., Ltd.) prior to development on X-ray film and photographic imaging to visualize the results. The sample size was 4 .

\section{Rat fracture model}

Male SD rats weighing approximately $180 \mathrm{~g}$ to $220 \mathrm{~g}$ were anesthetized with $10 \%$ chloral hydrate. The right lower limbs were depilated with hair removal cream and disinfected with $2 \%$ iodophor and $75 \%$ alcohol. A longitudinal incision was made from the medial part $3 \mathrm{~mm}$ below the tibial platform to the medial malleolus at 10 $\mathrm{mm}$, and the surface fascia at the incision site was cut. The tibia was dissected (Fig. 1a) and then cut in the middle with a wire clamp (Fig. 1b). The process required care to avoid damaging the fibula. The fracture area was washed with iodine and confirmed to be fully aligned (Fig. 1c). Then, $10 \mu \mathrm{L}$ PBS or $3 \times 10^{6}$ cells (dissolved in $10 \mu \mathrm{L}$ PBS) were injected into the fracture sites of the control, BM-MSC, and B-BM-MSC groups with a 1-mL syringe. Then, the surface fascia and skin were sutured, the incision was wrapped with sterile gauze and fixed with plaster, and the treatment outcome was evaluated at subsequent time points. The sample size was 6 .

\section{Observation of the fracture healing process}

At 2, 4, and 6 weeks after the operation, the fracture healing process was observed by computed tomography (CT) imaging, whereas visual inspection and hematoxylin and eosin $(\mathrm{H} \& \mathrm{E})$ staining were used to evaluate the fracture healing process at weeks 4 and 6 after the operation. The sample size was 6 . 


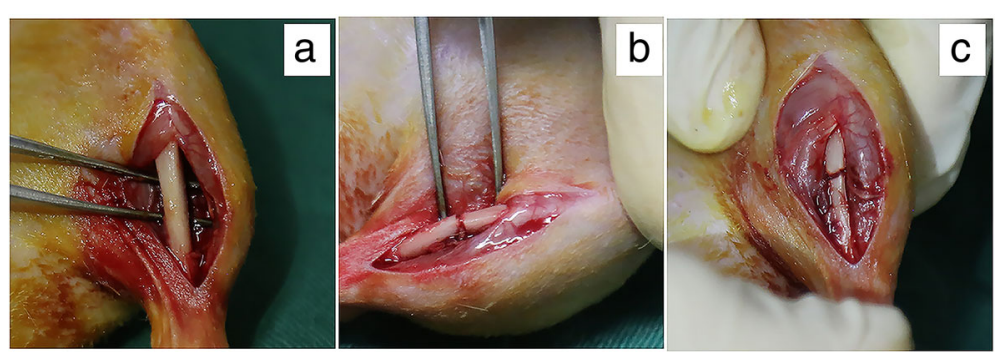

Fig. 1 Rat bone fracture model construction. a The tibia was dissected with blunt tweezers. $\mathbf{b}$ The tibia was cut in the middle. $\mathbf{c}$ The fracture was well aligned

\section{Statistical analysis}

The results are expressed as the mean \pm standard deviation. Prism 5.0 (GraphPad Software Inc., San Diego, CA, USA) was used for statistical analysis. Statistical comparisons among different groups were performed with oneway or two-way analysis of variance (ANOVA). $p<0.05$ indicated a statistical significance.

\section{Results}

The morphological features of B-BM-MSCs and BM-MSCs The early-stage BM-MSCs (Fig. 2a, b) were polygonshaped and round, while the early-stage B-BM-MSCs (Fig. 2e, f) were spindle-shaped, triangle-shaped, and polygon-shaped; some cells were clustered, and others were round and scattered. Both BM-MSCs and B-BMMSCs tended to become fusiform or streamlined (Fig. 2c, g) over time. The primary BM-MSCs reached 80 to $90 \%$ confluence at days 14 to 16 , while the primary B-BMMSCs reached $80-90 \%$ confluence at days 9 to 11 . However, mature BM-MSCs and B-BM-MSCs shared similar morphology (Fig. 2d, h) after GFP lentiviral transfection, and both adopted a fusiform or streamlined shape.

\section{MSC-associated marker expression in B-BM-MSCs and BM-MSCs}

According to the flow cytometry results, CD90 (99.18\% $\pm 0.15 \%)$, CD44 (98.47\% \pm 0.89\%), and CD29 (99.39\% $\pm 0.36 \%)$ were prominently expressed (> 97\%) in B-BM-MSCs, whereas CD106 (0.11\% $\pm 0.03 \%)$, CD45 (1.58\% $\pm 0.31 \%)$, and CD31 $(0.23 \% \pm 0.02 \%)$ were barely expressed $(<2 \%)$ in B-BM-MSCs. These results were consistent with the results of BM-MSCs. These results indicated that B-BMMSCs were successfully separated and cultured. It could be concluded that all the obtained MSCs were of high purity since MSC-associated markers were highly prevalent among these cells in every situation (Fig. 3).

\section{Proliferation ability of B-BM-MSCs and BM-MSCs in vitro} From 24 to $96 \mathrm{~h}$, the proliferative capacity of B-BMMSCs was higher than that of BM-MSCs (Fig. 4a); the corresponding cell proliferation rates on day 4 were $110.94 \% \pm 17.02 \%$ and $79.95 \% \pm 11.21 \%(p<0.05)$. In addition, the proliferative capacity of B-BM-MSCs was greater than that of BM-MSCs (Fig. 4b). These cells all showed the greatest proliferation ability from 72 to $96 \mathrm{~h}$.

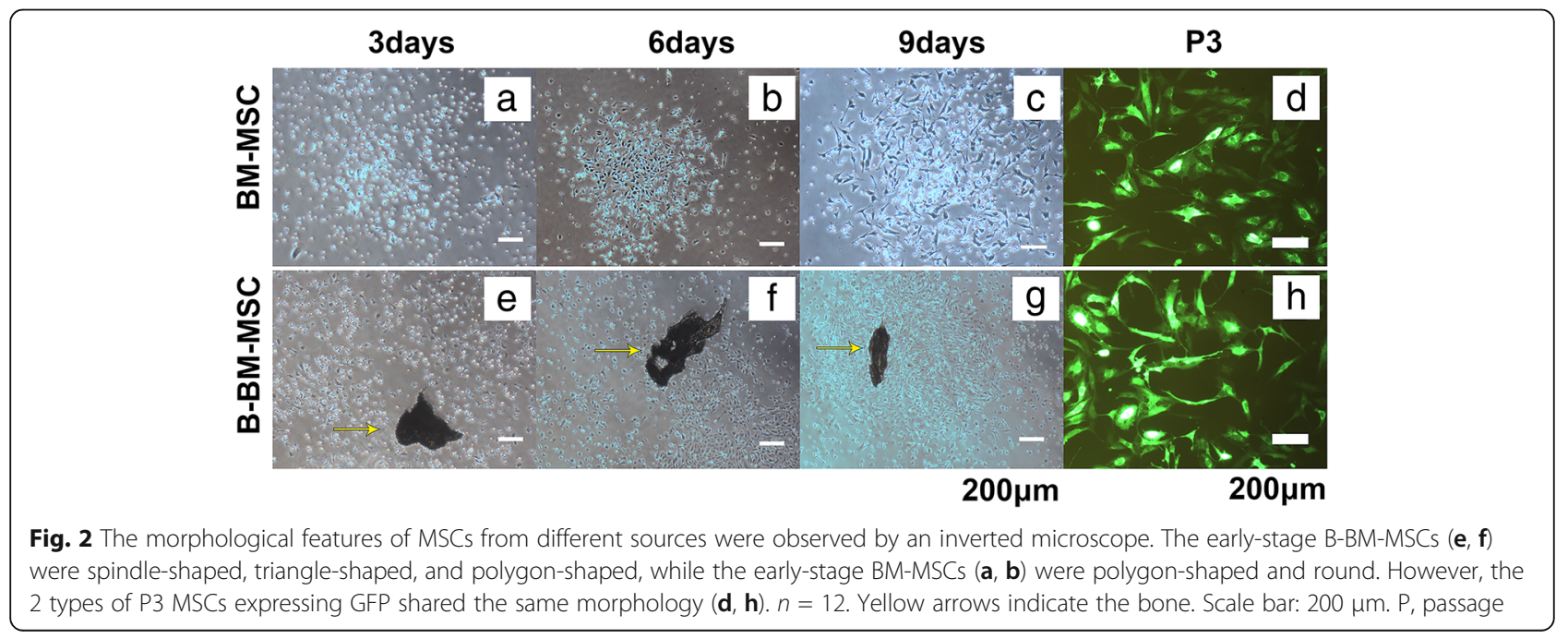



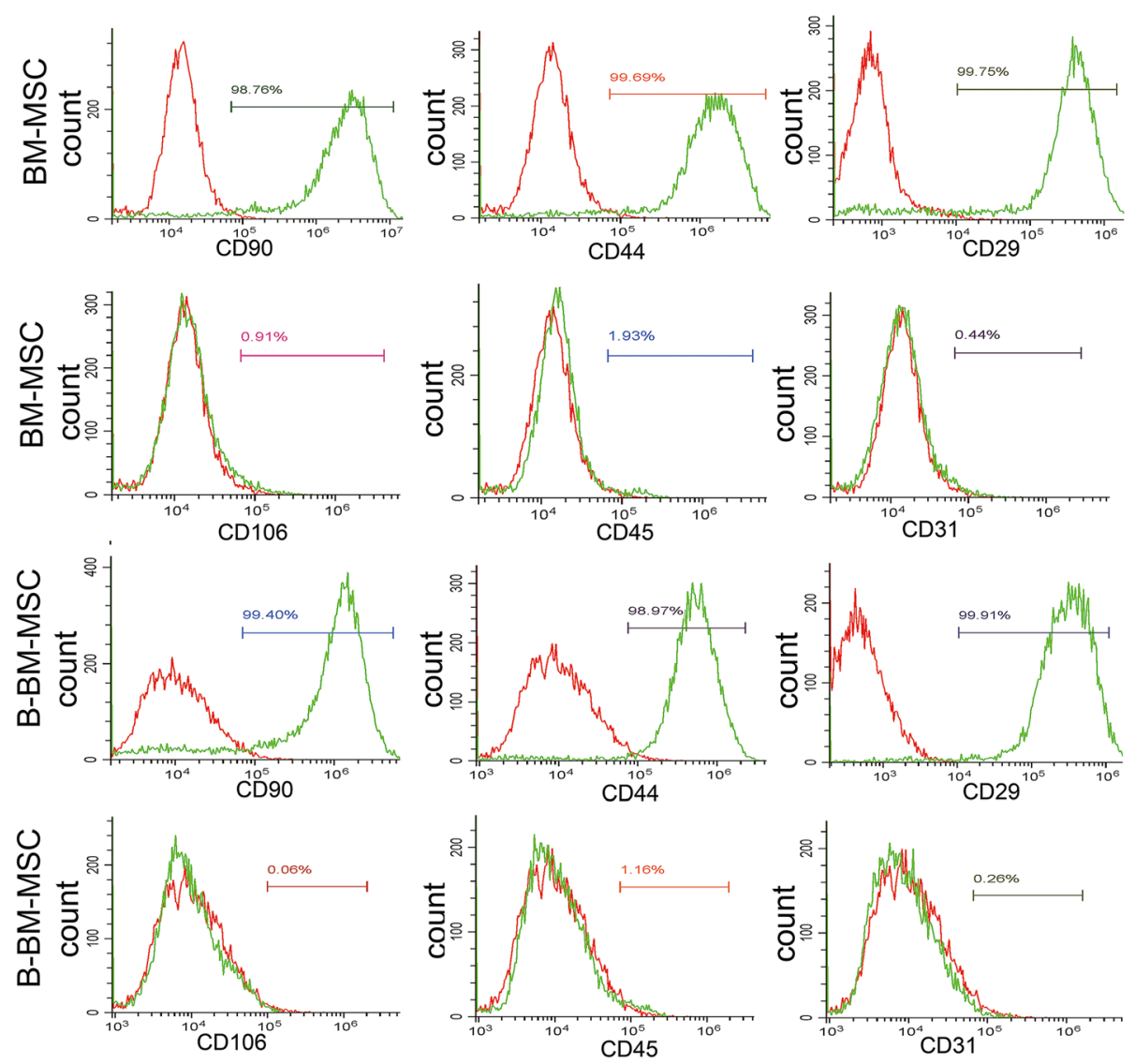

Fig. 3 Immunophenotyping of MSCs from different sources by flow cytometry assays. Two types of P3 MSCs were chosen for immunophenotyping. According to the flow cytometry results, CD90 (99.18\% \pm 0.15\%), CD44 (98.47\% $\pm 0.89 \%)$, and CD29 (99.39\% $\pm 0.36 \%)$ were prominently expressed (> 97\%), whereas CD106 $(0.11 \% \pm 0.03 \%), C D 45(1.58 \% \pm 0.31 \%)$, and CD31 $(0.23 \% \pm 0.02 \%)$ were barely expressed $(<2 \%)$ in B-BM-MSCs, which was consistent with the results of BM-MSCs. Stained cells are represented in green, whereas unstained cells are in red. $n=12$.
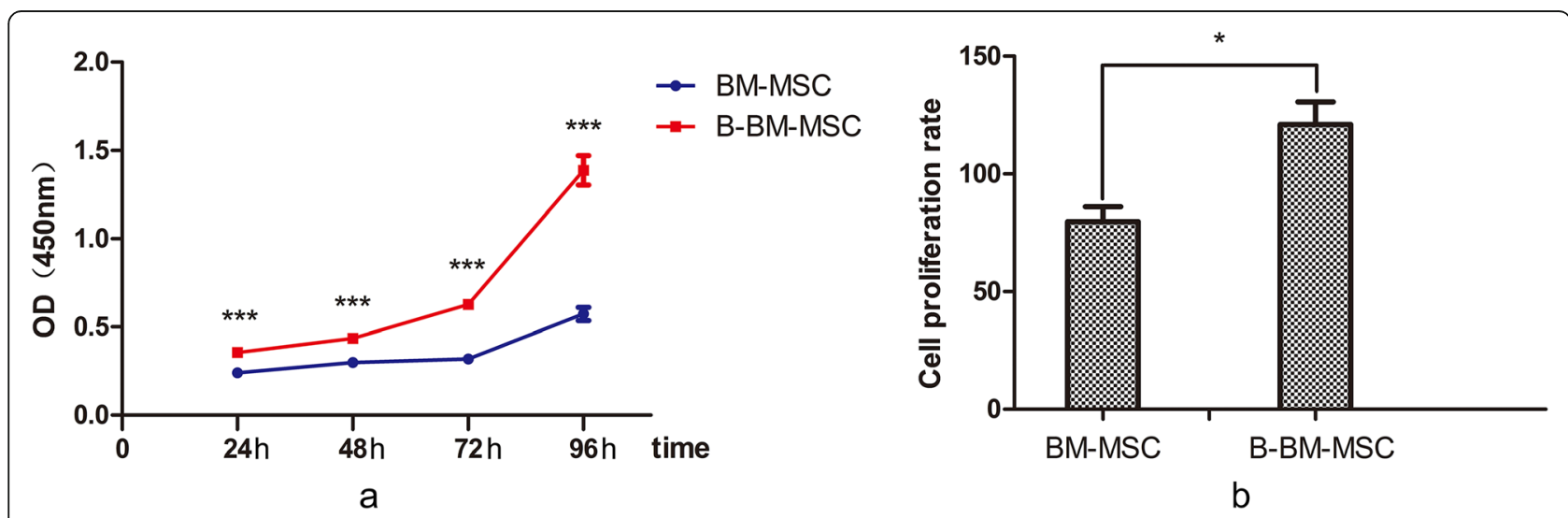

Fig. 4 The proliferation of MSCs from different sources was detected by CCK-8 assays. The proliferative capacity of B-BM-MSCs was greater than that of BM-MSCs, as the cell proliferation rates on day 4 were $110.94 \% \pm 17.02 \%$ and $79.95 \% \pm 11.21 \%$, respectively, $(p<0.05) . n=6 .{ }^{*} p<0.05,{ }^{* *} p<0.01$, ${ }^{* * *} p<0.001$ 


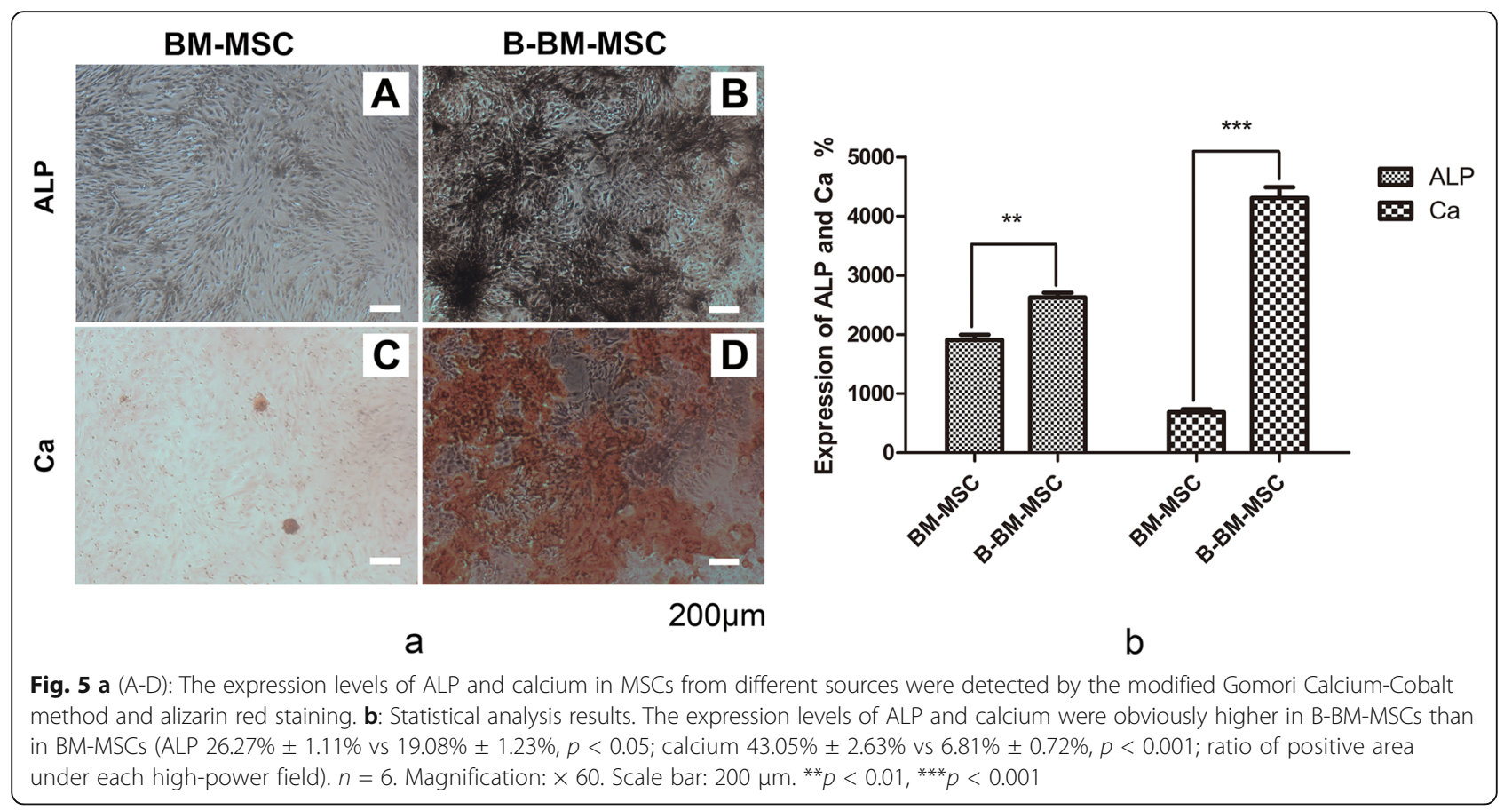

\section{Osteogenic differentiation ability of B-BM-MSCs and \\ BM-MSCs in vitro}

The results of alizarin red staining and the modified Gomori Calcium-Cobalt method showed considerable ALP expression and numerous calcium nodules after osteogenic induction for 14 days (Fig. 5a). The results showed that B-BMMSCs (RPA-HPT, $26.28 \% \pm 1.11 \%$ ) induced more ALPstained black plaques than BM-MSCs (RPA-HPT, 19.08\% \pm $1.23 \%)(p<0.05)$, and B-BM-MSCs generated more calcium nodules (RPA-HPT, $43.05 \% \pm 2.62 \%$ ) than BM-MSCs (RPAHPT, $6.81 \% \pm 0.72 \%)(p<0.001)$ (Fig. 5b). These findings showed that the osteogenic differentiation ability of BBM-MSCs was better than that of BM-MSCs in vitro.

\section{Expression of BMP-2 and TGF- $\beta 1$ in B-BM-MSCs and} BM-MSCs before and after osteogenic induction

Western blot results showed that the expression of both TGF- $\beta 1$ and BMP-2 was higher in B-BM-MSCs than in

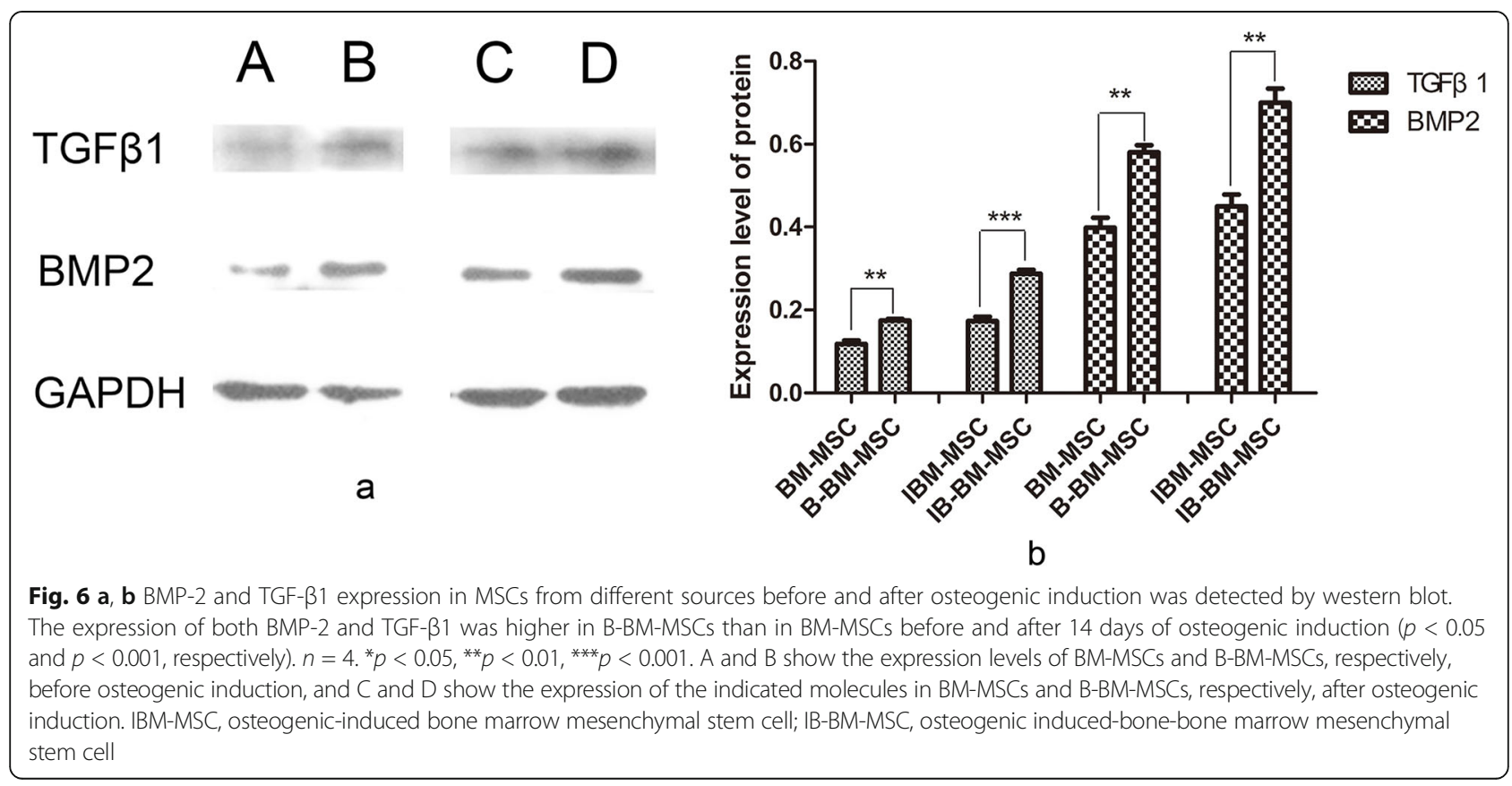



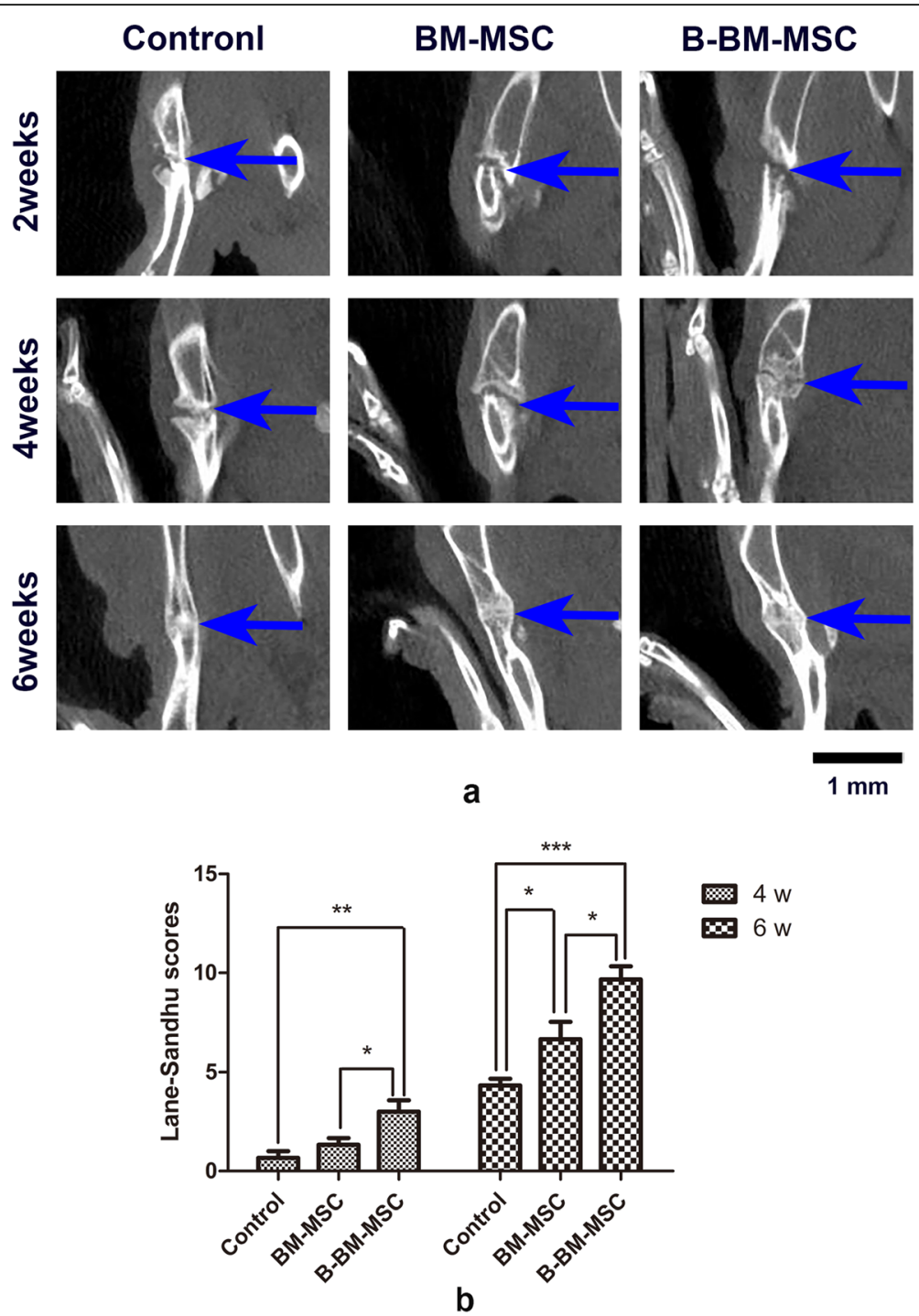

Fig. 7 a, $\mathbf{b}$ Healing in the rat fracture model by MSCs from different sources was detected by CT imaging. CT imaging showed that B-BM-MSCS had a stronger ability than BM-MSCs to promote fracture healing in vivo. Lane-Sandhu score analysis after the 4th and 6th weeks showed that B-BM-MSCs scored higher than BM-MSCs $(p<0.05) . n=6 .{ }^{*} p<0.05,{ }^{* *} p<0.01$, *** $p<0.001$

BM-MSCs before osteogenic induction. After a 2-week osteogenic induction, the expression of TGF- $\beta 1$ and BMP-2 remained higher in B-BM-MSCs compared to BM-MSCs (Fig. 6). This finding further confirmed that the osteogenic potential of B-BM-MSCs was greater than that of BM-MSCs in vitro.

\section{CT imaging observation of bone fracture healing}

In the 2nd week after the operation, the control, BMMSC, and B-BM-MSC groups all showed little callus formation at the bone fracture sites, while the number of calluses increased in week 4 after the operation in all 3 groups. In the 6 th week after the operation, there was substantial new bone formation in the B-BM-MSC group, and the medullary cavity was recanalized, indicating good regeneration, while remodeling was not prominent in the control group or the BM-MSC group (Fig. 7a). LaneSandhu score analysis (Fig. 7b) after the 4th and 6th weeks showed that B-BM-MSCs $(3.00 \pm 0.81$ and $9.67 \pm 0.94$, respectively) scored higher than BM-MSCs $(1.33 \pm 0.47$ and $6.67 \pm 1.25$, respectively) $(p<0.05)$. This finding indicated that B-BM-MSCs had a greater ability than BMMSCs to promote fracture healing in vivo.

\section{Gross observation of bone fracture repair}

To confirm our CT imaging findings, we collected bone specimens 4 and 6 weeks after construction of the rat 


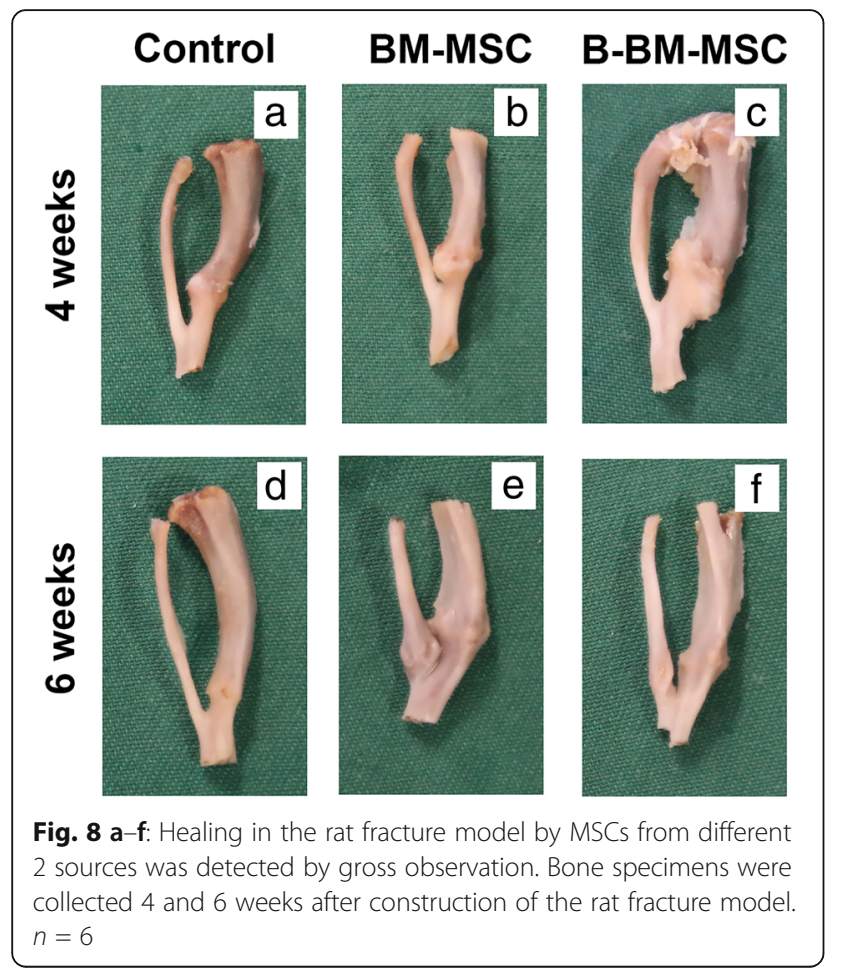

fracture model. In the control group, some new calluses formed around the fracture area 4 weeks after surgery (Fig. 8a). The fracture site showed on a large number of soft tissue connections, and the healing condition was not good enough. At the 6th week, the peripheral osteophytes had basically been absorbed, and the cortical bones were connected (Fig. 8d). The situation in the BM-MSC group (Fig. 8b, e) was slightly better than that in the control group, while the fracture healing in the B-BM-MSC group (Fig. 8c, f) at weeks 4 and 6 was significantly better than that in the control group. The sample size of this experiment was 6. Representative data from one animal per group are shown, and similar results were obtained for the other animals in each group. At the 4th and 6th weeks, fracture healing in the B-BM-MSC group was better than that in the BM-MSC group, while fracture healing in the BM-MSC group was better than that in the control group. These data indicated that both BM-MSCs and B-BM-MSCs can effectively promote fracture healing, although B-BM-MSCs produced superior results to BM-MSCs.

\section{Histological assessment of bone regeneration}

At the 4th week, large numbers of chondrocytes and osteogenic cells were observed in trabeculae, large populations of osteoblasts were observed around trabeculae, and hematopoietic cell proliferation in trabeculae was extremely active in the B-BM-MSC and BM-MSC groups (Fig. 9b, c). In the control group (Fig. 9a), some osteoblasts were also observed around trabeculae, whereas hyperplasia of chondrocytes and osteogenic cells was not obvious. At the 6th week, in the control (Fig. 9d) and BM-MSC groups (Fig. 9e), large numbers of osteoblasts were visible, and cells in trabeculae were tightly packed, whereas in the BBM-MSC group (Fig. 9f), trabeculae were mainly filled with hematopoietic cells, cells in the medullary cavity were loosely packed, and the trabeculae were more mature. The sample size of this experiment was 6 , and consistent results were obtained in other groups. At the 4th and 6th weeks, fracture healing and reconstruction were better in the BBM-MSC group than in the BM-MSC group, which showed better results than the control group in these 2 aspects. This finding further proved that B-BM-MSCs had a greater ability than BM-MSCs to promote fracture healing.

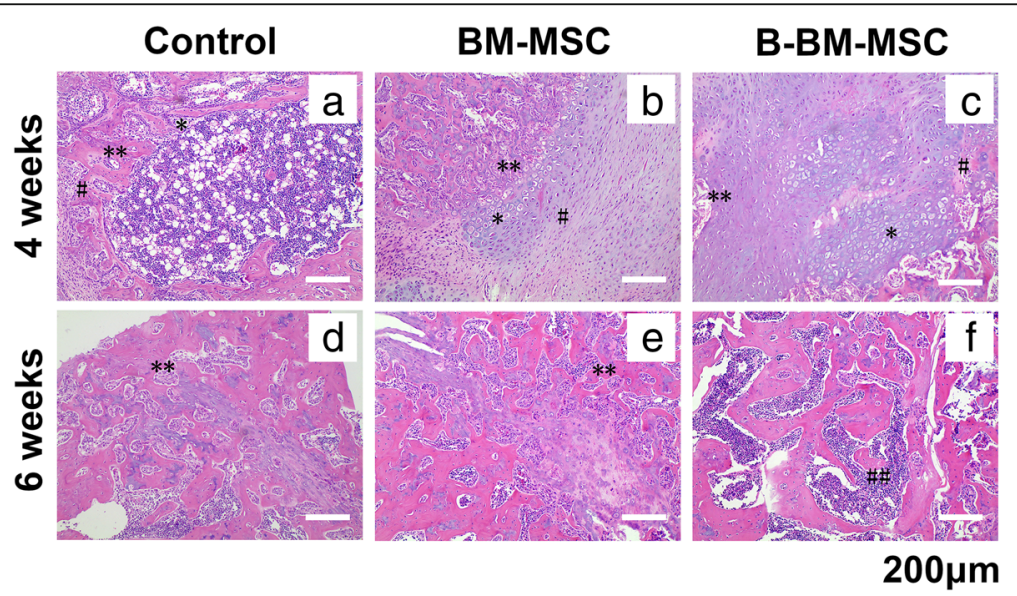

Fig. 9 a-f: Healing in the rat fracture model by MSCs from different sources was detected by H\&E staining. H\&E staining of bone specimens collected 4 and 6 weeks after construction of the rat fracture model. B-BM-MSCs had a stronger ability than BM-MSCs to promote fracture healing. $n=6 .{ }^{*}$ chondrocytes, \#osteogenic cells, **osteoblasts, \#\#hematopoietic cells 


\section{Discussion}

In this study, we obtained MSCs through the co-culture of bone and bone marrow for the first time and found that B-BM-MSCs have better proliferative, osteogenic differentiation, and fracture healing capacities than BMMSCs. We suggest that co-culturing the bone and bone marrow might be a useful method for obtaining seed cells for bone tissue repair.

Fernandez-Moure compared MSCs from the human bone and from the human bone marrow and concluded that CBF-MSCs had a weaker proliferative ability than BM-MSCs, but BM-MSCs had a significantly better osteogenic differentiation ability [13]. In terms of cell proliferation, we reached different conclusions than FernandezMoure. The reasons might be that MSCs were traditionally obtained through density gradient centrifugation, but some cancellous bone or soft tissue may be retained in this process [22]. For the isolation in this study, we used a filter with a diameter of $74 \mu \mathrm{m}$ to exclude cancellous bone and soft tissue. Alternatively, the discrepant results might be caused by different species used in our experiments. Daniel Blashki compared the proliferative ability of B-MSCs and BM-MSCs from rats [22], and our results are consistent with his conclusions. Concerning osteogenic differentiation, our results are similar to those reported by Fernandez-Moure in certain aspects.

Corradetti et al. demonstrated that the environment has an important effect on the differentiation direction of BMSCs and BM-MSCs [14], and some research has shown that the environment also somewhat determines the differentiation trend of MSCs [14, 23-25]. We suggest that the change in the environment of MSCs co-cultured with the bone and bone marrow resulted in improved proliferative and osteogenic differentiation abilities. TGF- $\beta 1$ promotes the differentiation of precursor osteoblasts in the early stage [20, 26], and BMP-2 is important in osteogenic differentiation and indispensable for the osteogenic differentiation of MSCs [21, 27]. The results of this study showed that B-BM-MSCs had higher TGF- $\beta 1$ and BMP-2 expression than BM-MSCs. We concluded that the coculture of the bone and bone marrow might enhance the osteogenic potential by increasing the expression of TGF$\beta 1$ and BMP-2 in MSCs. Wang et al. found that the upregulation of TGF- $\beta$ promoted tendon-to-bone healing after anterior cruciate ligament reconstruction with BM-MSCs [28], which further supports our conclusion.

The acquisition of B-MSCs can cause secondary damage to patients in clinical applications [13], while BM-MSCs can be easily obtained through bone marrow biopsy. Therefore, obtaining B-BM-MSCs through co-culture of a small amount of the bone with a relatively large amount of the bone marrow may avoid serious secondary damage to patients and ensure that the obtained MSCs have good proliferative activity and osteogenic potential.
Although the benefits of transplanting MSCs to promote fracture healing have been confirmed, there are still some problems remaining to be solved in clinical applications. Vadala et al. found that B-MSC injection in degenerated intervertebral disks in rats might induce osteophyte formation [29]. Thus, it is important to explore ways of inducing the localized differentiation of transplanted cells. In this study, we found that coculture of tissues from different sources could induce the directional differentiation of MSCs to some extent, but this did not completely solve this problem. In addition, we found that using 3 pieces of $3 \mathrm{~mm} \times 3 \mathrm{~mm}$ bone in 6-cm Petri dishes was helpful, but it is still unclear whether there is a better proportion of the bone in the culture system. We found that TGF-1 and BMP-2 in the co-culture of the bone and bone marrow played regulatory roles in promoting the proliferation, osteogenic differentiation, and fracture healing of MSCs, but the specific mechanism still needs further research. Therefore, further investigations are required to develop more satisfactory ways to use MSC transplantation to promote fracture healing.

\section{Conclusion}

In this study, we propose a novel way to obtain MSCs by co-culturing the bone and bone marrow from SD rats; these MSCs shared the same morphologic features and MSC-associated markers as traditional BM-MSCs, while their proliferative capacity and osteogenic potential were higher, and they successfully promoted fracture healing after injection into the fracture site. Therefore, this method may provide a promising source of MSCs for bone tissue engineering and clinical fracture treatment.

\section{Abbreviations}

ALP: Alkaline phosphatase; B-BM-MSCs: Bone-bone marrow mesenchymal stem cells; bFGF: Basic fibroblast growth factor; BM-MSCs: Bone marrow mesenchymal stem cells; BMP-2: Bone morphogenetic protein-2; BMSCs: Bone mesenchymal stem cells; CBF-MSCs: Mesenchymal stem cells from cortical bone fragments; CCK-8: Cell counting kit-8; CT: Computed tomography; DMEM: Dulbecco's modified Eagle's medium; FBS: Fetal bovine serum; GAPDH: Glyceraldehyde-3-phosphate dehydrogenase; GFP: Green fluorescent protein; HE: Hematoxylin and eosin; MSCs: Mesenchymal stem cells; OD: Optical density; P: Passage; PBS: Phosphate-buffered saline; PMSF: Phenylmethanesulfonyl fluoride; RIPA: Radioimmunoprecipitation assay; RPA-HPT: Ratio of positive area under each high-power field; Runx2: Runt-related transcription factor 2; SD: Sprague Dawley; SDSPAGE: Sodium dodecyl sulfate-polyacrylamide gel electrophoresis; Smads: Mothers against decapentaplegic homologs; TGF- $\beta 1$ : Transforming growth factor beta 1

\section{Acknowledgements \\ We acknowledge the great support and technical consultation of the Organ Transplantation Institute of Xiamen University, Central Laboratory of Medical College of Xiamen University, and Laboratory Animal Center of Xiamen University. Additionally, we would like to express our thanks to Ms. Haiyun Wang for her hard work at translating this article.}

\section{Authors' contributions}

CZ carried out cell isolation and differentiation, flow cytometry, CCK-8 assays, western blotting, rat fracture model experiments, histological studies, data 
analysis and interpretation, and drafting of the manuscript and provided final approval of the manuscript. MS and $\mathrm{HJ}$ performed cell expansion and differentiation, alizarin red and ALP staining, histological interpretation, rat fracture model experiments, and data assembly. $J \mathrm{~L}$ and $\mathrm{WL}$ participated in the rat fracture model experiments and $C T$ imaging. $W L$ participated in cell expansion and differentiation, flow cytometry, and CCK-8 assays. XC participated in the osteogenic differentiation induction and alizarin red and ALP staining. ZD participated in the overall design of the study and interpretation of data and helped edit the manuscript for intellectual and scientific content. GH conceived the overall project and scope, coordinated participants in the work, and aided in the interpretation of data and drafting of the manuscript. All authors read, made edits as necessary, and approved the final draft.

\section{Funding}

This work was funded by the Natural Science Foundation of Fujian Province of China (Project ID 2016J05208), Military Youth Training Program of China (Project ID 19QNP046), Scientific Research Projects on Military Logistics (Project ID CNJ16C013), and Youth Training Program of 18th Sub-region, Nanjing Military Region (Project ID 18FBQN2015006).

\section{Availability of data and materials}

The datasets used and/or analyzed during the current study are available from the corresponding author on reasonable request.

\section{Ethics approval and consent to participate}

This article does not contain any studies involving human participants. A total of 60 healthy male SD rats (aged 6 8 weeks, weighing 180 220 g) were obtained from the Laboratory Animal Center of Xiamen University. All rats were in good condition and had no related diseases according to examinations. This study was approved by the Ethics Committee of the Affiliated Southeast Hospital of Xiamen University and was conducted in strict accordance with the standards of the National Institutes of Health Guide for the Care and Use of Laboratory Animals.

\section{Consent for publication}

Not applicable

\section{Competing interests}

The authors declare that they have no competing interests.

\section{Author details}

'Center for Orthopedics, Affiliated Southeast Hospital of Xiamen University/ 909th Hospital of People's Liberation Army, 269 Zhanghua Middle Road, Zhangzhou 363000, Fujian Province, China. ${ }^{2}$ Xiamen University Medical College, Xiang'an South Road, Xiang'an District, Xiamen 361102, Fujian Province, China.

Received: 4 February 2019 Accepted: 23 August 2019 Published online: 03 September 2019

\section{References}

1. Einhorn TA, Gerstenfeld LC. Fracture healing: mechanisms and interventions. Nat Rev Rheumatol. 2015;11(1):45-54. https://doi.org/10.1038/nrrheum.2 014.164.

2. Pivonka P, Dunstan CR. Role of mathematical modeling in bone fracture healing. Bonekey Rep. 2012;1:221. https://doi.org/10.1038/bonekey.2012.221.

3. Arvidson $K$, Abdallah $B M$, Applegate $L A$, et al. Bone regeneration and stem cells. J Cell Mol Med. 2011;15(4):718-46. https://doi.org/10.1111/j.1582-4 934.2010.01224.x.

4. Fayaz HC, Giannoudis PV, Vrahas MS, Smith RM, Moran C, Pape HC, et al. The role of stem cells in fracture healing and nonunion. Int Orthop. 2011; 35(11):1587-97. https://doi.org/10.1007/s00264-011-1338-z.

5. Akpancar S, Tatar O, Turgut H, Akyildiz F, Ekinci S. The current perspectives of stem cell therapy in orthopedic surgery. Arch Trauma Res. 2016;5(4): e37976. https://doi.org/10.5812/atr.37976.

6. Marcucio RS, Nauth A, Giannoudis PV, et al. Stem cell therapies in orthopaedic trauma. J Orthop Trauma. 2015;29(Suppl 12):S24-7. https://doi. org/10.1097/BOT.0000000000000459.

7. Gao Y, Zhang Y, Lu Y, et al. TOB1 Deficiency Enhances the effect of bone marrow-derived mesenchymal stem cells on tendon-bone healing in a rat rotator cuff repair model. Cell Physiol Biochem. 2016;38(1):319-29. https:// doi.org/10.1159/000438632.

8. Maxson S, Lopez EA, Yoo D, Danilkovitch-Miagkova A, Leroux MA. Concise review: role of mesenchymal stem cells in wound repair. Stem Cells Transl Med. 2012;1(2):142-9. https://doi.org/10.5966/sctm.2011-0018.

9. Chanda D, Kumar S, Ponnazhagan S. Therapeutic potential of adult bone marrow-derived mesenchymal stem cells in diseases of the skeleton. J Cell Biochem. 2010;111(2):249-57. https://doi.org/10.1002/jcb.22701.

10. Ding DC, Chang YH, Shyu WC, Lin SZ. Human umbilical cord mesenchymal stem cells: a new era for stem cell therapy. Cell transplantation. 2015;24(3): 339-47. https://doi.org/10.3727/096368915X686841.

11. El Omar R, Beroud J, Stoltz JF, Menu P, Velot E, Decot V. Umbilical cord mesenchymal stem cells: the new gold standard for mesenchymal stem cell-based therapies? Tissue eng Part B, Rev. 2014;20(5):523-44. https://doi. org/10.1089/ten.TEB.2013.0664.

12. Hisha H, Nishino T, Kawamura M, Adachi S, Ikehara S. Successful bone marrow transplantation by bone grafts in chimeric-resistant combination. Exp Hematol. 1995;23(4):347-52.

13. Fernandez-Moure JS, Corradetti B, Chan P, et al. Enhanced osteogenic potential of mesenchymal stem cells from cortical bone: a comparative analysis. Stem Cell Res Ther. 2015;6. https://doi.org/10.1186/S13287-015-0193-Z.

14. Corradetti B, Taraballi F, Powell S, et al. Osteoprogenitor cells from bone marrow and cortical bone: understanding how the environment affects their fate. Stem Cells Dev. 2015;24(9):1112-23. https://doi.org/10.1089/scd.2014.0351.

15. Berebichez-Fridman R, Gomez-Garcia R, Granados-Montiel J, et al. The Holy Grail of orthopedic surgery: mesenchymal stem cells-their current uses and potential applications. Stem Cells Int. 2017. https://doi.org/10.1155/2017/2638305.

16. Strioga M, Viswanathan S, Darinskas A, Slaby O, Michalek J. Same or not the same? Comparison of adipose tissue-derived versus bone marrow-derived mesenchymal stem and stromal cells. Stem Cells Dev. 2012;21(14):2724-52. https://doi.org/10.1089/scd.2011.0722.

17. Farrell MJ, Fisher MB, Huang AH, Shin Jl, Farrell KM, Mauck RL. Functional properties of bone marrow-derived MSC-based engineered cartilage are unstable with very long-term in vitro culture. J Biomech. 2014;47(9):2173-82 https://doi.org/10.1016/j.jbiomech.2013.10.030.

18. Leyh M, Seitz A, Durselen $L$, et al. Subchondral bone influences chondrogenic differentiation and collagen production of human bone marrow-derived mesenchymal stem cells and articular chondrocytes. Arthritis Res Ther. 2014;16(5):453. https://doi.org/10.1186/s13075-014-0453-9.

19. Yamachika $E$, Tsujigiwa $H$, Matsubara $M$, et al. Basic fibroblast growth factor supports expansion of mouse compact bone-derived mesenchymal stem cells (MSCs) and regeneration of bone from MSC in vivo. J Mol Histol. 2012; 43(2):223-33. https://doi.org/10.1007/s10735-011-9385-8.

20. Vlacic-Zischke J, Hamlet SM, Frus T, Tonetti MS, Ivanovski S. The influence of surface microroughness and hydrophilicity of titanium on the up-regulation of TGF beta/BMP signalling in osteoblasts. Biomaterials. 2011;32(3):665-71. https://doi.org/10.1016/j.biomaterials.2010.09.025.

21. Rahman MS, Akhtar N, Jamil HM, Banik RS, Asaduzzaman SM. TGF-beta/BMP signaling and other molecular events: regulation of osteoblastogenesis and bone formation. Bone Res. 2015;3:15005. https://doi.org/10.1038/boneres.2015.5.

22. Blashki D, Murphy MB, Ferrari M, Simmons PJ, Tasciotti E. Mesenchymal stem cells from cortical bone demonstrate increased clonal incidence, potency, and developmental capacity compared to their bone marrowderived counterparts. J Tissue Eng. 2016;7 doi: Unsp 204173141666119610. 1177/2041731416661196.

23. Trivanovic D, Kocic J, Mojsilovic S, et al. Mesenchymal stem cells isolated from peripheral blood and umbilical cord Wharton's jelly. Srp Ark Celok Lek. 2013;141(3-4):178-86. https://doi.org/10.2298/Sarh1304178t.

24. Zhang H, Ma X, Zhang L, Guan XM, Bai T, Xue CH. The ability to form cartilage of NPMSC and BMSC in SD rats. Int J Clin Exp Med. 2015;8(4):4989-96.

25. Huang C, Dai J, Zhang XA. Environmental physical cues determine the lineage specification of mesenchymal stem cells. Biochimica et biophysica acta. 2015;1850(6):1261-6. https://doi.org/10.1016/j.bbagen.2015.02.011.

26. Buttner M, Moller $\mathrm{S}$, Keller M, et al. Over-sulfated chondroitin sulfate derivatives induce osteogenic differentiation of hMSC independent of BMP-2 and TGF-beta1 signalling. J Cell Physiol. 2013;228(2):330-40. https://doi.org/10.1002/jcp.24135.

27. Wu MR, Chen GQ, Li YP. TGF-beta and BMP signaling in osteoblast, skeletal development, and bone formation, homeostasis and disease. Bone Res. 2016;4. doi: Artn 16009 https://doi.org/10.1038/Boneres.2016.9.

28. Wang $\mathrm{R}, \mathrm{Xu} \mathrm{B}, \mathrm{Xu} \mathrm{HG}$. Up-regulation of TGF-beta promotes tendon-to-bone healing after anterior cruciate ligament reconstruction using bone marrow- 
derived mesenchymal stem cells through the TGF-beta/MAPK signaling pathway in a New Zealand white rabbit model. Cell Physiol Biochem. 2017; 41(1):213-26. https://doi.org/10.1159/000456046.

29. Vadala G, Sowa G, Hubert M, Gilbertson LG, Denaro V, Kang JD.

Mesenchymal stem cells injection in degenerated intervertebral disc: cell leakage may induce osteophyte formation. J Tissue Eng Regen Med. 2012; 6(5):348-55. https://doi.org/10.1002/term.433.

\section{Publisher's Note}

Springer Nature remains neutral with regard to jurisdictional claims in published maps and institutional affiliations.

Ready to submit your research? Choose BMC and benefit from:

- fast, convenient online submission

- thorough peer review by experienced researchers in your field

- rapid publication on acceptance

- support for research data, including large and complex data types

- gold Open Access which fosters wider collaboration and increased citations

- maximum visibility for your research: over $100 \mathrm{M}$ website views per year

At BMC, research is always in progress.

Learn more biomedcentral.com/submissions 